\title{
Analysis of Predictive Factors Influencing Early Patency in Adult Autogenous Arterio Venous Accesses
}

\author{
S.K.Balaji ${ }^{1}$,S.Prathap Kumar $^{2}$,S.Jeyakumar ${ }^{3}$,N.Sritharan ${ }^{4}$,P.Ilayakumar ${ }^{5}$ \\ 1,2,3,4,5 (Institute of Vascular Surgery, Madras Medical College, India)
}

\begin{abstract}
Arteriovenous(AV) accesses are the lifeline for Chronic kidney disease (CKD) patients and providing a durable access is a challenge for every vascular surgeon. The maturation of autogenous AV accesses at 4-6 weeks is the biggest hurdle more than the creation of $A V$ access. There are many traditional preoperative predictors for Arteriovenous fistula ( AVF) maturation including old age female sex, obesity, smoking, presents of atherosclerotic risk factors like Coronary Artery Disease(CAD), Cerebrovascular Disease(CVD), Peripheral Vascular Disease(PAD), Diabetes Mellitus. In this study in addition to the evaluation of the above risk factors routine duplex was done to see additional parameters like artery diameter reactive hyperemia, and venous diameter, venous distensibility and to rule out central venous stenosis. Preoperative mean arterial pressure and blood pressure between the two upper limbs were also taken into consideration after thorough physical examination. A new concept of spiral laminar flow detected by post op duplex was also included in this study. With all the parameters and routine postoperative antiplatelets with clopidogrel $75 \mathrm{mg}$ once daily in all patients the influence of such factors on early patency of adult autogenous AV accesses were assessed at 2 weeks post procedure.
\end{abstract}

Keywords: AVF maturation, Reactive hyperaemia,Spiral laminar flow

\section{Introduction}

CKD patients have various comorbidities like old age, systemic hypertension, diabetes mellitus, coronary artery disease, cerebrovascular accidents, peripheral arterial disease, obesity all of which serve as the biggest hurdles for AVF maturation. These factors contribute to early failure of AVF's which occurs within first 3 months (20$50 \%$ ).But 3 to 4 months is a long duration and if the patency of AVF can be determined by predictors in the preoperative, intraoperative and post-operative period (early patency of AVF) possibly within 10 to 14 days these patients could be subjected to procedures to salvage failing fistula or to create a new access at an appropriate alternative site.Preoperative duplex ultrasound for vein mapping has been used selectively in ESRD. But routine use of duplex for evaluating arterial pathologies and hemodynamic assessments will help a lot in predicting early patency because most of these patients have native arterial disease per se [1].Smoking is one another risk factor which may unmask or worsen the peripheral arterial disease and is an important determinant of the presence of inadequate inflow into the fistula. The intake of antihypertensive drugs during the fistula creation and the timing of AV access creation has been a matter of debate and have been evaluated. The intake of antihypertensives on the day of surgery has not been an important factor as long as the preoperative mean arterial pressure is maintained above $85 \mathrm{mmHg}$. The timing of AV access creation is important and studies have shown that AVF created before initiation of hemodialysis creates a favourable atmosphere for AVF maturation. Certain other factors like preoperative urea and creatinine do not seem to affect early patency and interestingly low haemoglobin levels might have a paradoxical effect on early AVF patency by its effect on haemoxygenase and increased nitric oxide. Antiplatelets given in the immediate post-operative period may not have a role in maturation but may have a role in the early patency of AV accesses by mitigating the rate of early thrombosis (Dialysis access consortium trial)

One another important post-operative duplex follow up finding is the spiral laminar flow measured on post-operative day (POD) 1 and 7 which prognosticates patients regarding patency and maturation. In fact as the days progress a good functioning AV access would have a good thrill, systolic broadening and spiral laminar flow which has high positive predictive values. None of the studies have evaluated the early patency of AV accesses with regard to pre-operative, intra operative and post-operative predictive factors. The advantage of such a study would be to identify and possibly modify such risk factors and predict the early patency or thrombosis /failure of such fistulas so that the patient need not wait for another 4-6 weeks for maturation which would drastically reduce the morbidity and costs to the patient.

\section{Aims And Objectives}

To comprehensively analyse the predictive factors influencing early patency of adult arteriovenous accesses at 2 weeks post operatively in CKD patients 


\section{III.Materials And Methods}

In this study, all CKD patients were evaluated first with history and physical examination with proximal tourniquet and looking for the tap in the vein, identifying side branches arterial pulsations and Allen's test in the non-dominant forearm. They were subjected to routine duplex ultra sound with $7 \mathrm{MHz}$ probe for evaluation of arteries and veins with above mentioned parameters. Routine hemoglobin, urea, creatinine, platelet count were done and preoperative Mean Arterial Pressure was maintained $>85 \mathrm{mmHg}$. Arteries and veins to be anastomosed were preoperatively marked and fistula created in end to side fashion using 7.0 prolene and standard techniques. Postoperative antiplateletswere started in all patients at a dose of $75 \mathrm{mg}$ once daily. Spiral laminar flow was studied postoperatively and the data were analyzed with regard to the influence of these predictors in the early patency at two weeks of these AV fistulas. The sample size was 100, with the data collected both retrospectively and prospectively, after obtaining proper informed consent from the patients.

\subsection{Study Type\& Period}

Retrospective, prospective Duration: 2015 to 2016.

\subsection{Inclusion Criteria}

All adult CKD patients enrolled for primary AV access creation

3.3 Exclusion Criteria

Redo AV accesses, pediatric AV accesses, post-transplant failure patients enrolled for AV access

\section{Discussion}

Advanced age and decreased life expectancy results in poor outcomes, especially in forearm radio cephalic accesses, reasons being increased incidence of heart failure, stiffness of arteries, atheroma, calcifications and increased hemodynamic compromise [2].Female sex has been associated with poor outcomes in the radio cephalic fistulas (RCAVF).Reasons are small caliber vessels, poor venoreactivity, hormonal, hypercoagulable factors. In case of the diabetic population, there appears to be distal diffuse disease and calcifications which results in AVF failures.The primary AVF failures in one study were $51 \%$ in diabetics when compared to $41 \%$ in non-diabetics in RCAVF [2]. The problems with obesity are deep veins, difficult cannulation. AVF outcomes appear to be favourable in the setting of preoperative duplex Smoking compounds the inherent native arterial disease in patients with CKD. It has a primary role in causing endothelial dysfunction [3],creating a hypercoagulable, prothrombotic state and producing vasoconstriction and vasospasm. The presence of coronary artery disease, cerebrovascular accidents and peripheral arterial disease will compound the failures of the AVF that is created because atherosclerosis is a systemic disease. In addition to these factors CKD patients have native arterial disease due to intimal hyperplasia, medial calcifications.

Antiplatelets like thienopyridines have to be stopped prior to surgery to prevent the bleeding complications. Anticoagulants such as warfarin have to be stopped before 5 days of surgery at least and a PT/INR should be evaluated before embarking on surgery. The routine use of antihypertensives has not been considered a negative factor in determining the potential for early thrombosis because as long as the MAP remained $>85 \mathrm{mmHg}$ and there is no preoperative hypotension, such drugs have minimal say on the patency.It has been found that post-operative antiplatelets given for a minimum period of 2 weeks might not affect maturation but it might have a positive influence on early patency [4]. Low hemoglobin because of the effects on haemoxygenase and nitric oxide may paradoxically improve the patency of AVF's by altering the hemodynamics and the wall shear stress.Platelet counts are very essential for three reasons; 1 .Patient may be on heparin dialysis which would have decreased the platelets 2.Post op patients may need to be started on anticoagulation or antiplatelets and thus a baseline value is essential,3.In the presence of normal platelet counts, a bleeding tendency in the immediate post-operative period might indicate an acquired qualitative platelet dysfunction(Bright's Disease) which is common in CKD patients [5].

The inflow artery must provide adequate blood without rendering the hand ischemic. The subclavian, axillary, brachial, radial and ulnar pulses are palpated in both arms. A BP difference of greater than $20 \mathrm{mmHg}$ is considered significant.Allen's test is performed in all patients to see the dominant artery in the forearm and hand and the adequacy of the palmar arch [6].Venous system examination should be examined with a tourniquet, blood pressure cuff which is inflated to a pressure of $60 \mathrm{mmHg}$ or $5 \mathrm{mmHg}$ above the diastolic pressure and left in place for 5 minutes [6]. But physical examination is subjective and the rate of successful AVF creation based on physical examination alone is around $72 \%$.Hence Kidney Disease Outcome Quality Initiative (KDOQI) has recently recommended that noninvasive imaging should also be added routinely to physical examination and that it should be the standard of care.

Preoperative duplex parameters to be seen for veins are the vein diameter, venous distensibility, length of the segment of vein without branches, for the arteries are artery diameter, pressure and velocity waveforms, reactive hyperemia, stenosis and calcifications [7]. KDOQI guidelines have suggested a minimal vein diameter of 2.5 mmfor successful AVF maturation [8]. Similarly, Silva et al demonstrated that a minimum $2.5 \mathrm{~mm}$ vein 
diameter was required [9], but Mendez et al had improved outcomes even with $2.2 \mathrm{~mm}$ diameter [10]. An adequate length of the cephalic or the basilic vein in a straight course without side branches would be ideal for AVF outcomes .A superficial vein within $6 \mathrm{~mm}$ from the skin surface would be ideal for maturation and cannulation. Tributaries which are found within $5 \mathrm{~cm}$ of the proposed site of anastamosis should be preoperatively identified and marked and ligated intraoperatively to improve the early patency. Malorvh found that venous distensibility was predictive of outcomes [11]. Successful AVFs showed a mean percentage dilatation of $44 \%$ compared with only $11 \%$ in the unsuccessful AVF group. Silva et al also suggested that a minimum venous distensibility of at least $2.5 \mathrm{~mm}$ achieved with a proximal tourniquet decreased the early failure rates to $8 \%$ and improved the primary patency to $81 \%$.KDOQI guidelines have given a minimal diameter criteria for radial arteries at $2 \mathrm{~mm}$.

There are no fixed diameter criteria for the brachial artery because these arteries are sufficiently larger even in thin females to keep an AV access patent [8]. Reactive hyperemia is an useful adjunct in the radial artery evaluation especially if they are doubtful with regard to size and caliber .If the difference in the peak systolic velocity is greater than $5 \mathrm{~cm} / \mathrm{s}$ after fist clinching and opening that means that the radial artery is ideal for the creation of the AV access [2]. Preoperative preparations are done and the mean arterial pressure is kept at $>85$ mmHg.By transverse plane, low velocity settings, colour Doppler, SLF (spiral laminar flow) will be imaged as a REDBLUE SHIFT.A loss of the spiral laminar flow pattern would suggest either an arterial inflow disease or an anastamotic problem or venous outflow disease. In many trials, the venous outflow stenosis detection by SLF has been shown to be the best earliest predictor of early patency of AV accesses [8, 12, 13].

\section{Observationandresults}

Table 1: Influence of Preoperative Predictors in AV Accesses

\begin{tabular}{|c|c|c|}
\hline Age & $<65$ & $>65$ Years \\
\hline $\mathbf{N}(\mathbf{1 0 0})$ & 93 & 7 \\
\hline Lt Ref & 65 & 2 \\
\hline Others & 28 & 5 \\
\hline Sex & Male & Female \\
\hline $\mathbf{N}(100)$ & 75 & 25 \\
\hline Lt Ref & 54 & 13 \\
\hline 0thers & $21(28 \%)$ & $12(48 \%)$ \\
\hline Site Of Fistula & Right & Left \\
\hline Radiocephalic(Rcf) & 6 & 67 \\
\hline Brachiocephalic(Bcf) & 4 & 21 \\
\hline Brachiobasilic(Bbf) & 1 & Nil \\
\hline Ulnarbasilic(Ubf) & Nil & 1 \\
\hline Diabetes Mellitus & Left Rcf & Others \\
\hline $\mathrm{N}=21$ & 12 & $9(42 \%)$ \\
\hline Hypertension & Left Rcf & Others \\
\hline $\mathrm{N}=48$ & 31 & $17(35 \%)$ \\
\hline Cad/Cva & Left Rcf & Others \\
\hline $\mathrm{N}=4$ & 1 & $3(75 \%)$ \\
\hline Pvd & Left Rcf & Others \\
\hline $\mathbf{N}=3$ & 2 & $1(33 \%)$ \\
\hline Bmi & Lt Ref & Lt Bcf \\
\hline $\mathbf{N}=1$ & Nil & 1 \\
\hline Smoking & Lt Ref & Others \\
\hline $\mathrm{N}=\mathbf{1 0}$ & 5 & $5(50 \%)$ \\
\hline
\end{tabular}

$\mathrm{RCF}$-radiocephalic fistula,CAD-coronary artery disease,CVA-Cerebrovascular accidents,PVD-peripheral vascular disease, BMI- body mass index

In this study $93 \%$ of patients were under the age of 65 years and $7 \%$ were above 65 years . $71 \%$ of patients older than 65 years had an AV access other than radio cephalic fistula .In this study, $48 \%$ of females and $28 \%$ of males had an AV access other than radio cephalic fistula. $33 \%$ of patients received an AV access other than the routine radio cephalic fistulas $67 \%$ underwent left RCF,42\% of diabetics had an AV access other than radio cephalic fistulas and $35 \%$ of hypertensives received an AV access other than radio cephalic fistulas. $75 \%$ of patients with CAD/CVA had an AV access other than radio cephalic fistula. 33\% of patients with PVD had an AV access other than radio cephalic fistulas. There was only one patient with BMI $>35 \mathrm{Kg} / \mathrm{m}^{2}$ and she had received a left brachiocephalic fistula. In this study $50 \%$ of smokers had an AV access other than radio cephalic fistula.

Table 2: Distribution of patients based on catheter $(\mathrm{N}=100)$ On Catheter 


\begin{tabular}{|l|l|}
\hline Not On Catheter & 61 \\
\hline
\end{tabular}

The patients in this study were taken up for AVF creation on an elective basis regardless of the presence or absence of catheters and these patients had a CVC inserted from a minimum of 5 days duration to a maximum of 2 months duration. Those patients who had edema of their arms at the site of fistula creation were requested to undergo one more session of dialysis to reduce the edema.

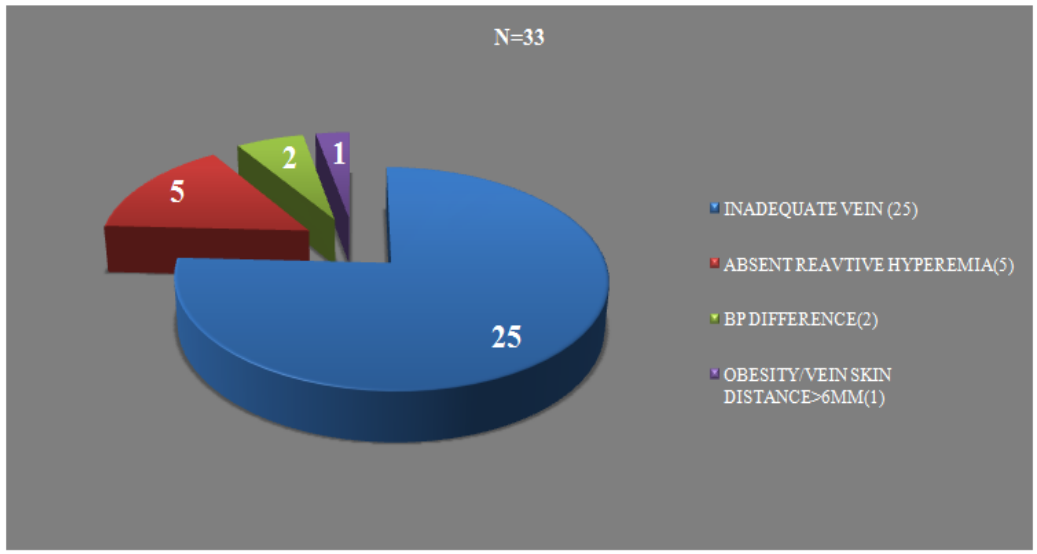

Fig 1: Causes of Alternate Site AV Access Selection

In the study 33 patients received an $\mathrm{AV}$ access other than the routine left $\mathrm{RC}$ fistulas. Inadequate veins were the most common cost in 25 patients absent reactive hyperemia in 5 patients, BP difference between both upper limbs in 2 patients and obesity with increased vein distance from the skin in 1 patient.

Table 3: Lab Investigations And Blood Pressure (BP)

\begin{tabular}{|c|c|c|c|}
\hline & MINIMUM & MAXIMUM & MEAN \\
\hline Haemoglobin & 2.9 & 13.4 & 9.077 \\
\hline Urea & 41 & 220 & 94.972 \\
\hline Creatinine & 2.3 & 21.5 & 5.938 \\
\hline Preoperative Bp & $110 / 70$ & $160 / 90$ & 138/92 Mmhg \\
\hline
\end{tabular}

We have found in this study that preoperative hemoglobin levels had no influence on the early patency of AV access. Preoperative creatinine values ranged from 2.3 to $21.5 \mathrm{mg} / \mathrm{dl}$ and urea values of the range of 41 to $220 \mathrm{mg} / \mathrm{dl}$, still this wide range of values did not influence the outcomes. In this study, the patients who were on anti hypertensives were not stopped from taking their routine antihypertensives if the mean pressure was more than $85 \mathrm{~mm} \mathrm{Hg}$. The range of blood pressures was from110/70 to160/90 $\mathrm{mmHg}$.

Table 4: Pre And Post-Operative Duplex Criteria

\begin{tabular}{|c|c|c|c|c|}
\hline & $\begin{array}{l}\text { Radial } \\
\text { (Min) }\end{array}$ & Brachial & Positive & Negative \\
\hline Artery Diameter & $1.6 \mathrm{Mm}$ & 3.5 Mm & 100 & 0 \\
\hline Vein & $2.2 \mathrm{Mm}$ & $\begin{array}{l}2.2 \mathrm{Mm} \\
\text { (Cephalic) }\end{array}$ & 100 & 0 \\
\hline Vein Distensibility & $>2.5 \mathrm{Mm}$ & $>2.5 \mathrm{Mm}$ & 100 & 0 \\
\hline Vein Length 6cm & & & 100 & 0 \\
\hline $\begin{array}{l}\text { Distance From Skin } 6 \\
\text { Mm }\end{array}$ & & & 99 & 1 \\
\hline $\begin{array}{l}\text { Side Branches Within } \\
5 \mathrm{Cm}\end{array}$ & & & 95 & 5 \\
\hline Reactive Hyperemia & & & 95 & 5 \\
\hline Central Vein Stenosis & & & 100 & 0 \\
\hline $\begin{array}{l}\text { SPIRAL LAMINAR } \\
\text { FLOW }\end{array}$ & & & 98 & 2 \\
\hline
\end{tabular}

In this study, a minimum radial artery diameter of $1.6 \mathrm{~mm}$ was adequate for maintaining early patency. A brachial artery diameter of $3.5 \mathrm{~mm}$ was adequate although in literature suggests that the diameter of the brachial cephalic vein was a better predictor than brachial artery diameters.Even though KDOQI guidelines suggest a minimum diameter of $2 \mathrm{~mm}$ for the radial arteries and $2.5 \mathrm{~mm}$ for the veins for successful AVF maturation in Indian population, in this study, we have found that a minimum radial artery diameter of $1.6 \mathrm{~mm}$ and a minimum vein diameter of $2.2 \mathrm{~mm}$ were only required for the early patency of AV access. One important finding is that even with smaller venous diameters, venous distensibility of greater than $2.5 \mathrm{~mm}$ with a pressure 
cuff applied proximally and inflated to $60 \mathrm{mmHg}$ pressure was an important predictor of early patency. In this study, all the patients were selected based on a vein length of at least $6 \mathrm{~cm}$, within $6 \mathrm{~mm}$ from skin surface except for the one patient, who was obese and hence had a deeper vein and a brachiocephalic fistula was done instead of radio cephalic fistula.

In 95 of the cases, a side branch was identified either with pre op duplex or intra operatively and was routinely ligated. In this study, 95 patients had reactive hyperemia test in their radial arteries positive, with 5 patients exhibiting negative results which led to the creation of AV access at alternative sites other than left radio cephalic fistulas. All the patients in this study were screened for central vein stenosis/thrombosis and none had positive findings.In this study, the spiral laminar flow was monitored on day 1 and day 7 and found that red blue shift was present in 98 cases and 2 cases spiral laminar flow was absent. We have also found that the laminar flow replaces the turbulence flow length and can be considered as the best immediate postop predictor of early patency. All patients were prescribed T.clopidogrel $75 \mathrm{mg}$ OD for the two weeks up to suture removal and none of the patients had bleeding complications which necessitated the withdrawal of the drug, In this 96 patients had their AV accesses patent at 2 weeks postop 2 patients lost follow up and there were 2 failures and both these failures were in females. There were some limitations in this study because the hypercoagulable state was not evaluated in these patients and infection was not ruled out with blood cultures. Statistical significance could not be derived because it was a small group of patients and randomization was not done.

\section{Conclusion}

The site of AV accesses does not affect the early patency. In the presence of atherosclerotic risk factors (diabetes, hypertension, CAD, CVA, PVD,obesity,smoking,old age), consideration should be given for a duplex guided proximal arm AV access as a first choice. Female sex appears to be an independent risk factor for early thrombosis even in duplex selected cases. The addition of hypercoagulable work up and infection screening might help in such cases. The presence or absence of catheter does not affect early thrombosis even at 2 months in this study. Low haemoglobin values do not affect early patency possibly due to increase in haemoxygenase and nitric oxide and favourable shear stress. There seems to be no cut off values for urea creatinine in predicting early patency of AV access. Preoperative(mean arterial pressure MAP maintained greater than $85 \mathrm{~mm} \mathrm{Hg}$ is desirable and the antihypertensive medications seem to have no effect on early patency. In INDIAN population a minimum arterial diameter of $1.6 \mathrm{~mm}$, vein diameter of $2.2 \mathrm{~mm}$ could be ideal for early patency. Venous distensibility of $>40 \%$ or minimum $2.5 \mathrm{~mm}$ on tourniquet would be essential. Vein length of $6 \mathrm{~cm}$, distance from skin $<6 \mathrm{~mm}$ prerequisites. Novel duplex techniques like reactive hyperemia should be routinely used in the preop assessment of arteries used in AVF creation. Spiral laminar flow is an excellent tool for early detection of fistula patency and its probable maturation potential. Post op antiplatelets can be used at least for 2 weeks with minimal bleeding risk. Its effect on early patency could be assessed with further case control studies

\section{References}

[1]. Alik Farber, Peter B Imrey, Thomas S Huber, James M Kaufman, Larry W Kraiss, Brett Larive, Liang Li, Harold I Feldman,Multiple Pre-Operative and Intra-Operative Factors Predict Early Fistula Thrombosis in the Hemodialysis Fistula Maturation Study, Journal of Vascular Surgery, 63 (1), 2016 163-70 e6

[2]. Martin Michael Ferring, An investigation into factors predicting patency and maturation of arteriovenous fistulae used for haemodialysis in end stage renal disease, University of Birmingham, Edgbaston, Birmingham, B15 2TT, United Kingdom, 2012

[3]. JohnWHallett, Joseph L Mills,Comprehensive vascular and endovascular surgery, (John F.Kennedy Blvd, PA: Elsevier Saunders, 2009)

[4]. RaghavWusirika, PandurangaRao,Effect of Clopidogrel on Arteriovenous Fistulas for Dialysis, JAMA 300(14), 2008 1647-1649

[5]. Cronenwett and Johnston, Rutherford's vascular surgery, (John F.Kennedy Blvd, PA: Elsevier Saunders, 2014)

[6]. Huber TS, Ozaki CK, Flynn TC, Lee WA, Berceli SA, Hirneise CM, Carlton LM, Carter JW, Ross EA, Seeger JM,Prospective validation of an algorithm to maximize native AVF for chronic HD access, Journal of Vascular Surgery, 36(3), 2002452-9

[7]. Wesley S Moore,Vascular and Endovascular Surgery,(John F.Kennedy Blvd, PA: Elsevier Saunders, 2013)

[8]. KDOQI guidelines, 2006 recommendations

[9]. Silva MB Jr, Hobson RW, Pappas PJ, Jamil Z, Araki CT, Goldberg MC, Gwertzman G, Padberg FT Jr, A strategy for increasing use of autogenous hemodialysis access procedures: impact of preoperative noninvasive evaluation, Journal of Vascular Surgery, 27(2), 1998 302-307

[10]. Mendes RR, Farber MA, Marston WA, Dinwiddie LC, Keagy BA, Burnham SJ, Prediction of wrist arteriovenous fistula maturation with preoperative vein mapping with ultrasonography, Journal of Vascular Surgery, 36(3), 2002 460-463

[11]. Marlovh, Vascular access for hemodialysis: arteriovenous fistula, Therapeutic Apheresis and Dialysis, 9(3), 214-217

[12]. SVS-Arterivenous haemodialysis access, Journal of vascular surgery,2008

[13]. AneeshSrivastava, Varun Mittal, HiraLal, TarunJavali, NiteshPatidar, SanjoySureka, ShikharAggarwal, Spiral laminar flow - The earliest predictor for maturation of arteriovenous fistula for hemodialysis access, Indian Journal of Urology, 31(3), 2015 240-244 